\title{
Do patients over 40 years of age benefit from surgical closure of atrial septal defects?
}

\author{
M Jemielity, W Dyszkiewicz, L Paluszkiewicz, B Perek, P Buczkowski, A Poniżyński
}

\begin{abstract}
Objective-To determine the value of surgical closure of atrial septal defects in patients over 40 years of age.

Methods-Retrospective analysis of 76 patients (63 women, 13 men), age range 40-62 years (mean (SD) 45.8 (5.1) years), who underwent surgical repair of atrial septal defect. Pre- and postoperative clinical status (New York Heart Association (NYHA) functional class) was assessed, and ECG, $x$ ray, and echocardiographic investigations performed. Follow up was between 1 and 17 years.

Results-One operative and one late death occurred during the study period. Before operation, 47 patients $(61.8 \%)$ were in NYHA functional classes III and IV. After operation, 61 patients $(82.4 \%)$ were in classes I and II. Four patients had atrial fibrillation before surgery versus nine after surgery. Before operation, 52 patients had intensified pulmonary vascularity compared with only seven after operation. Echocardiographic examination showed a significant reduction in right ventricular dimension $(4.10(0.91) v 2.95(0.36) \mathrm{cm}, \mathrm{p}<0.001)$. No residual intracardiac shunts were identified on echocardiographic follow up.

Conclusions-Surgical closure of atrial septal defects in patients over 40 years old can improve their clinical status and prevent right ventricular dilatation and insufficiency.

(Heart 2001;85:300-303)
\end{abstract}

Keywords: atrial septal defect; cardiac surgery

Atrial septal defect is the most common congenital heart lesion found in adult life. It is detected in about $10 \%$ of heart malformations at birth and in almost $40 \%$ of those present in adults. ${ }^{1}$ Closure of the majority of atrial septal defects, especially in patients who are symptomatic with large left to right shunts, is recommended in children and young adults because of the low operative risk. ${ }^{2}$ Furthermore, complete surgical correction at that time avoids subsequent congestive heart failure, pulmonary hypertension, thromboembolic complications, and arrhythmias, and thereby increases life expectancy. ${ }^{34}$

Atrial septal defects go unrecognised for years in many patients because of the absence or subtle nature of clinical symptoms. ${ }^{5}$ Unlike the known beneficial effects in young patients, the benefits of closure in adults over 40 years old remain controversial and a clear policy on the medical management of such patients has not been established. Some investigators have claimed that surgical repair in these patients, as
In the present study we therefore evaluated the long term results of surgical repair of atrial septal defects in patients over 40 years of age.

\section{Methods}

PATIENTS

This study involved 76 patients operated on between 1981 and 1995. Seventy six (63 women and 13 men) underwent surgical repair of an isolated secundum atrial septal defect. The age range was 40-62 years, mean (SD) 45.8 (5.1) years. The diagnosis was established by cardiac catheterisation and cross sectional echocardiography in some patients, and after the introduction of Doppler and transoesophageal echocardiography, only by echocardiography in the remainder. In all patients coronary arteriography was performed before operation but no significant changes were found in the coronary arteries. The mean time between establishment of diagnosis and operation was 8.7 (12.4) years (range two months to 33 years).

Seventy four patients (97.4\%) were followed up for 1 to 17 years (mean 6.9). The clinical status (New York Heart Association (NYHA) functional class) and the findings on ECG, chest $x$ ray, and echocardiography (cross sectional and Doppler) were assessed.

The data were analysed by the paired $t$ test and McNemar's test. A probability value of $\mathrm{p}<0.05$ was considered significant.

SURGICAL PROCEDURE

All operations were performed using cardiopulmonary bypass and moderate total body hypothermia of between $27-28^{\circ} \mathrm{C}$. The chest was opened by a median sternotomy. After total cardiopulmonary bypass had been \footnotetext{
compared with medical treatment, increases long term survival and limits the deterioration in circulatory function. ${ }^{6}$ Shah and colleagues, analysing a group of 34 medically treated and 48 surgically treated patients, found no significant differences in survival rates or symptoms between the two groups during follow up. ${ }^{7}$ As surgical procedures are obviously associated with mortality (approximately 3.3\%), ${ }^{8}$ and with prolonged hospital stay, increased costs, the need for blood transfusion, and often psychological trauma, it is important for both the patient and the doctor to analyse the long term results of surgical treatment.

Correspondence to: mjemielity@poland.com

Accepted 11 September 2000

term results of surgical treatment.

Department of
Cardiac Surgery,

Institute of Cardiology
Karol Marcinkowski

University of Medical

M Jemielity

P Buczkowski
} 


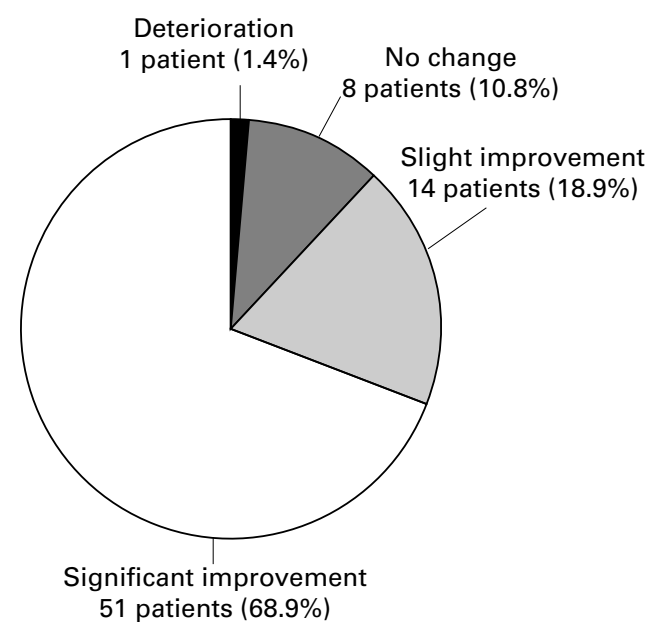

Figure 1 Patients' assessment of changes in their clinical status after operation.

Table 1 Distribution of clinical symptoms among the 88 patients

\begin{tabular}{llc}
\hline Symptoms & $\begin{array}{l}\text { Before correction } \\
(n)\end{array}$ & $\begin{array}{l}\text { After correction } \\
(n)\end{array}$ \\
\hline Easy fatigue & 68 & $26^{\star}$ \\
General weakness & 57 & $5^{\star}$ \\
Syncope & 17 & $4^{\star}$ \\
Exertional dyspnoea & 55 & $22^{\star}$ \\
Rest dyspnoea & 11 & $1^{\star}$ \\
Palpitations & 63 & $16^{\star}$ \\
\hline
\end{tabular}

${ }^{\star} \mathrm{p}<0.05 v$ before correction.

instituted, cold crystalloid cardioplegic solution (St Thomas' Hospital formula) was given into the root of the aorta. The method used to close the defect depended on its size and anatomical type. Seventy patients with an isolated atrial septal defect underwent direct suture closure. In only six patients was the defect considered large enough to require an autologous pericardial patch. The patch was sutured with double layers of Prolene 5-0 continuous suture. In addition to the primary atrial septal defect closure, 10 patients underwent DeVega annuloplasty for tricuspid regurgitation.

\section{Results}

FOLLOW UP EXAMINATIONS

Clinical status improved in 65 patients and deterioration was noted in only one case. Clinical status at follow up is outlined in fig 1 . The most common complaints before operation were easy fatigue and palpitations, expressed by over $70 \%$ of the patients. Only four patients

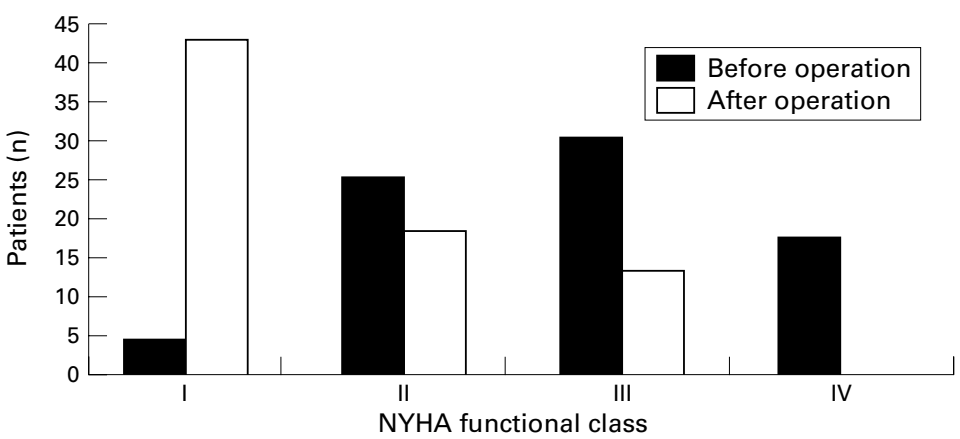

Figure 2 New York Heart Association functional class before and after correction.
Table 2 Drugs given

\begin{tabular}{llc}
\hline & $\begin{array}{l}\text { Before operation } \\
(n)\end{array}$ & $\begin{array}{l}\text { After operation } \\
(n)\end{array}$ \\
\hline Diuretics & 24 & 5 \\
Antiarrhythmics & 22 & 31 \\
Anticoagulants & 2 & 9 \\
\hline
\end{tabular}

were asymptomatic. After operation the total number of reported symptoms decreased considerably (table 1). Before operation, 47 patients $(61.8 \%)$ were in NYHA functional classes III and IV. Following surgical correction, 61 patients $(82.4 \%)$ were in classes I and II (fig 2).

Two thromboembolic events (one ischaemic stroke with permanent neurological dysfunction, and one transient ischaemic attack) were observed at follow up. One patient with primary closure of an atrial septal defect underwent reoperation for a recurrence of the defect in the early postoperative period.

Antiarrhythmic drugs were being taken by 22 patients (29\%) before operation, compared with 31 patients $(42 \%)$ after operation. The need for anticoagulant treatment also increased after surgical repair (table 2).

Four patients had atrial fibrillation before operation, and this number increased to nine after operation. Before operation, 66 patients had incomplete and three complete right bundle branch block (RBBB), 30 patients showed ECG signs of right ventricular overload and hypertrophy, 10 had non-specific changes of the ST-T segment, six had single ectopic ventricular beats, six had ectopic atrial beats, and one had the Wolff-Parkinson-White syndrome. Postoperatively, right ventricular hypertrophy decreased (6 (8\%) v 30 (39\%) before surgery), as did right axis deviation (5 $v 17$ ). Postoperatively, non-specific changes of the ST-T segment increased from 10 to 14 . Atrial flutter was present in two patients following surgery, and sick sinus syndrome in one.

Chest $x$ ray (postero-anterior and side projections) revealed significant improvements in pulmonary vascularity and reduction in the size of the right ventricle. Preoperatively, 52 patients had intensified pulmonary vascularity, but after surgery this was present in only seven. Pulmonary trunk prominence was seen in 58 patients before surgery and in nine afterwards. The cardiac volume index (CVI) was calculated before and after surgery from the radiographic views, but no significant changes were found (mean (SD) 589 (134) $\mathrm{ml} / \mathrm{m}$ before $v 552$ (106) $\mathrm{ml} / \mathrm{m}$ after correction).

Cross sectional echocardiography showed that the right ventricular dimension had decreased from $4.10(0.91) \mathrm{mm}$ before to 2.95 $(0.36) \mathrm{mm}$ after surgical treatment $(\mathrm{p}<0.001)$. The left ventricular end diastolic dimension increased from $3.92(0.31) \mathrm{mm}$ before to $4.28(0.45) \mathrm{mm}$ after operation, but the difference was not significant. Values of other cardiac indices are summarised in table 3. Before surgery, Doppler echocardiography showed tricuspid insufficiency in 37 patients, with a mean right ventricular systolic pressure of 43.5 (6.4) $\mathrm{mm} \mathrm{Hg}$ (range 29.1- 
Table 3 The basic cross sectional echocardiographic variables of the heart chambers and walls

\begin{tabular}{lll}
\hline Variable & $\begin{array}{l}\text { Before operation } \\
(\mathrm{mm})\end{array}$ & $\begin{array}{l}\text { After operation } \\
(\mathrm{mm})\end{array}$ \\
\hline Ao & $2.59(0.34)$ & $2.72(0.29)$ \\
LA & $3.14(0.65)$ & $3.23(0.68)$ \\
LVED & $3.92(0.31)$ & $4.28(0.45)$ \\
LVES & $2.65(0.44)$ & $3.13(0.57)$ \\
RV & $4.10(0.91)$ & $2.95(0.36)^{\star}$ \\
IVS & $1.02(0.14)$ & $0.96(0.12)$ \\
LVPW & $0.92(0.23)$ & $0.93(0.15)$ \\
PA & $2.63(0.52)$ & $2.42(0.48)$ \\
\hline
\end{tabular}

Values are mean (SD).

${ }^{\star} \mathrm{p}<0.05 \mathrm{v}$ before operation.

Ao, aortic root diameter; IVS, interventricular septal dimension; LA, left atrial dimension; LVED, left ventricular end diastolic dimension; LVES, left ventricular end systolic dimension; LVPW, left ventricular posterior wall dimension; PA, pulmonary artery diameter; RV, right ventricular dimension.

$69.5 \mathrm{~mm} \mathrm{Hg}$ ). Ten of these required DeVega annuloplasty. Postoperatively, only seven had mild insufficiency, with a mean right ventricular systolic pressure of 27.5 (4.3) $\mathrm{mm} \mathrm{Hg}$ (range 24.1-34.5 $\mathrm{mm} \mathrm{Hg}$ ). The maximum velocity of tricuspid diastolic flow decreased significantly after operation, from 91.1 (12.5) to $51.8(15.4) \mathrm{cm} / \mathrm{s}(\mathrm{p}<0.05)$. No residual intracardiac shunt was identified on echocardiographic follow up.

IN HOSPITAL AND LATE DEATHS

There were two deaths in the series, one in hospital and one late. The hospital death involved a 62 year old patient who was in NYHA class III/IV and in congestive heart failure with chronic bronchial asthma. Closure of his large atrial septal defect was performed using a pericardial patch. The immediate postoperative course was uneventful but on the seventh postoperative day severe bronchospasm occurred and two days later respiratory failure developed. Attempts to wean the patient from mechanical ventilation were unsuccessful and he died on the 20th postoperative day.

The late death was of a 66 year old woman who died four years after the operation. She was in NYHA class III preoperatively. The atrial septal defect was closed by direct suture and her postoperative course was satisfactory. She was last reviewed four months before her death. At that time she was in NYHA class I and her chest $x$ ray showed significant improvements in pulmonary vascularity and a diminution in right ventricular size. She died after severe retrosternal chest pain. Unfortunately, a necropsy examination was not performed, but her death was presumed to have been caused by myocardial infarction.

\section{Discussion}

During the first decade of life most patients with atrial septal defects are asymptomatic. Around $50 \%$ begin to complain of exertional dyspnoea by the age of 20 , and nearly $100 \%$ do so by the sixth decade. The natural history of atrial septal defect shows $75 \%$ mortality in patients over 50 years old and almost $90 \%$ mortality in those with an uncorrected defect over the age of $60 .{ }^{4}$ There is now a consensus view that surgical closure of these defects dur- ing or before the early 20 s ensures that long term survival is not significantly different from that of age and sex matched controls.

Throughout the 40 years over which surgical correction has been carried out, perioperative mortality has decreased from $12.5 \%$ in the late 1950 s to $6 \%$ in the 1960 s and to below $0.5 \%$ today. ${ }^{6}$ This major improvement in survival appears to be a reflection of both better operative technique and better postoperative care, so surgical closure of this relatively common anomaly has became widely recommended even for older patients. In spite of the very good results of surgery, even in patients with raised pulmonary artery pressure, several studies published in the past few years have questioned the policy of routine surgical closure in older patients. ${ }^{4{ }^{7-11}}$ Studies analysing the late outcome after surgical correction of atrial septal defects in adults over 40 years of age have been reported. ${ }^{8}{ }^{12-15}$ Age was found to be a significant and independent predictor of surgical mortality and morbidity at late follow up. ${ }^{16}$ Furthermore, Murphy and colleagues proved that patients undergoing surgery after the age of 40 years are at increased risk of postoperative cardiovascular complications, whereas children and young adults enjoyed an excellent prognosis. ${ }^{8}$ It was thought in the 1960s that surgical correction of atrial septal defects in adults over 45 was of benefit only in those with minimal symptoms, and that operative closure after 45 years of age was of questionable value. ${ }^{16}$ Later, Knight and Lenox pointed out that a moderate increase in pulmonary artery pressure before operation (up to $65 \mathrm{~mm} \mathrm{Hg}$ systolic) did not increase overall hospital mortality. ${ }^{14}$ They also claimed that operating on patients with severe pulmonary hypertension was too hazardous. However, the hospital mortality in our series confirms its safety, even in older patients. This is in agreement with the study by Konstantinides and colleagues, where the clinical course of surgically treated patients with isolated atrial septal defects, diagnosed after the age of 40, was compared with those who received only medical treatment. ${ }^{6}$ These investigators suggested that the surgical repair of atrial septal defects substantially increased long term survival in middle aged and elderly patients.

The postoperative course and long term results depend mainly on the patient's preoperative clinical status. It is known that pulmonary hypertension is always significant in patients with multiple cardiovascular symptoms. ${ }^{4}$ Impaired function before operation (NYHA classes III and IV) is one of the predictors of death. Also, in our series advanced NYHA classification had a pronounced impact on early and late mortality. The only patients who did not survive the operation or the follow up period were in functional class III or IV. Improvement by at least one functional class has also been reported in other studies. ${ }^{812-15}{ }^{17}$ In our study we found a significant improvement in functional status in approximately $70 \%$ of surgically treated patients (figs 1 and 2). However, Shah and colleagues detected no significant change in 
NYHA class assessed postoperatively in comparison with medical treatment, but all their patients were in NYHA classes I or II on entering the study. ${ }^{7}$

The frequency of atrial fibrillation in patients with atrial septal defects increases with age. ${ }^{8-1016}$ The number of patients with atrial fibrillation in our study increased at follow up despite evident clinical improvement, but was still low. Antiarrhythmic drugs were given to $42 \%$ of our patients, which may explain why the incidence of atrial fibrillation in our series was lower than reported elsewhere.$^{16}$ It is possible that the mechanisms leading to atrial rhythm disturbances are different before and after surgery. Preoperatively, atrial distension is the main cause of atrial fibrillation. In spite of the fact that ECG evidence of right ventricular overload decreased in our patients, from 39\% before to $8 \%$ after operation, the number of patients with atrial fibrillation actually increased. The precise mechanism of postoperative atrial fibrillation in patients with atrial septal defects is not known. ${ }^{15}$ It is suggested that it could be related to the formation of an ectopic focus at the site of surgical repair, or the resulting scar. There is also evidence that venous cannulation at the time of operative repair may contribute to the incidence of arrhythmias observed at long term follow up.

Atrial fibrillation is a possible reason for the increased incidence of thromboembolic events in patients operated on to correct an atrial septal defect. ${ }^{1315}$ Although the incidence of atrial fibrillation increased in our patients, the number of thromboembolic complications was relatively low $(3.7 \%)$. It is likely that anticoagulation treatment prevented more cerebrovascular events. Shah and colleagues reported a similar incidence of systemic embolism in both medically and surgically treated groups. ${ }^{7} \mathrm{We}$ suggest careful monitoring for arrhythmias after closure of atrial septal defects and also the use of anticoagulants to prevent thromboembolic events. Because of the risk of postoperative thromboembolism, we prefer direct suture closure of isolated atrial septal defects and the use of pericardial patches for large defects. Fiore and associates employed a Dacron or autologous pericardial patch for closure of defects in elderly patients. ${ }^{2}$ They claimed that direct suturing in the older population was more likely to distort the atrial septum and so foster arrhythmias. We avoided Dacron patches because of the increased thromboembolic risk associated with their use. Although routine anticoagulant treatment is not recommended after closure of an atrial septal defect, ${ }^{2}$ we gave anticoagulants for three months after operation - maintaining the international normalised ratio between 2.5 and 3.5-to all patients with a pericardial patch. Patients with persistent atrial fibrillation and those with a history of embolic events should always have anticoagulant treatment before and after surgical intervention.

\section{CONCLUSIONS}

The low mortality and morbidity in patients over 40 years of age who have been operated on for atrial septal defect is a strong argument in support of surgical versus medical treatment. Long term follow up has shown the beneficial effects of surgical correction in terms of improvements in clinical status (NYHA functional class), diminution of pulmonary vascularity (determined by chest $x$ ray), and a reduction in the size of the right heart (from echocardiographic measurements). Thus surgical treatment is recommended for older patients, even these over 60, who are in NYHA classes III and IV, though it requires meticulous postoperative care.

1 Dave KS, Pakrashi BC, Woolder GH, et al. Atrial septal defect in adults: clinical and hemodynamic results of defect in adults: clinical and herno

2 Fiore AC, Naunheim KS, Kessler KA, et al. Surgical closure of atrial septal defect in patients older than 50 years of age. of atrial septal defect in patien

3 Campbell M. Natural history of atrial septal defect. Br Heart f 1970;32:820-6.

4 Konstantinides S, Geibel A, Kasper W, et al. The natural course of atrial septal defect in adults-a still unsettled issue. Klin Wochenschr 1991;69:506-10.

5 Fahmy A, Schiavone W. Unusual clinical presentations of secundum atrial septal defect. Chest 1993;104:1075-8.

6 Konstantinides S, Geibel A, Olschewski $M$, et al. A comparison of surgical and medical therapy for atrial septal defect in adults. N Engl f Med 1995;333:469-73.

7 Shah D, Azhar M, Oakley CM, et al. Natural history of secundum atrial septal defect in adults after medical or surgical treatment: a historical prospective study. Br Heart 7 1994;71:224-8.

8 Murphy JG, Gersh BJ, McGoon MD, et al. Long term outcome after surgical repair of isolated atrial septal defects. Follow-up at 27 to 32 years. N Engl F Med 1990;323:164550

9 Cheng TO. The natural course of atrial septal defect in adults-a still unsettled issue. Clin Invest 1992;70:85.

10 Ward C. Secundum atrial septal defect: routine surgical treatment is not of proven benefit. Br Heart $\mathcal{F} 1994 ; 71: 219-$

11 Perloff JK. Surgical closure of atrial septal defect in adults. $N$ Engl f Med 1995;333:513-14.

12 Gault JH, Morrow AG, Gay WA, et al. Atrial septal defect in patients over the age of forty years. Circulation 1968;37: 261-71.

13 Richmond DE, Lowe JB, Barratt-Boyes BG. Results of surgical repair of atrial septal defects in the middle-aged and elderly. Thorax 1969;24:536-42.

14 Knight M, Lenox S. Results of surgery for atrial septal defect in patients of 40 years and over. Thorax 1972;27: 577-82.

15 Hairston P, Parker EF, Arrants JE, et al. The adult atrial septal defect: results of surgical repair. Ann Surg 1974;179: 799-803.

16 Sutton MG, Tajik AJ, McGoon DC. Atrial septal defect in patients aged 60 years or older: results and long-term postoperative follow-up. Circulation 1981;64:402-9.

17 Shibata Y, Abe T, Kuribayashi R, et al. Surgical treatment of isolated secundum atrial septal defect in patients more than 50 years old. Ann Thorac Surg 1996;62:1096-9. 